Pacific Journal of Mathematics

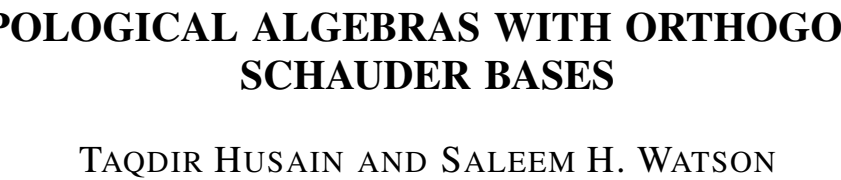




\title{
TOPOLOGICAL ALGEBRAS WITH ORTHOGONAL SCHAUDER BASES
}

\author{
TAQDiR HuSAIN AND SALEEM WATSON
}

\begin{abstract}
Topological algebras with Schauder orthogonal bases are studied. Radicals, closed ideals and closed maximal ideals of such algebras are described. It turns out that a locally $m$ convex algebra with identity and having an orthogonal basis is metrizable. This implies that a complete locally $m$-convex algebra with an orthogonal basis and identity is algebraically and topologically isomorphic with the Fréchet algebra of all complex sequences.
\end{abstract}

Introduction. Let $A$ be a topological algebra. A (Schauder) basis $\left\{x_{n}\right\}$ in $A$ is called an orthogonal (Schauder) basis if $x_{n} x_{m}=$ $\delta_{n m} x_{n}, \quad n, m=1,2, \cdots$ where $\delta_{n m}$ denotes the Kronecker delta. Algebras with such bases (actually a variation of this definition which we will discuss below) were first studied by Husain. In [3] Husain and Liang proved that every multiplicative linear functional on a Fréchet algebra (i.e., complete metrizable locally $m$-convex algebra) with an unconditional orthogonal basis is continuous. This result answers Michael's question [5] (as to whether every multiplicative linear functional on a Fréchet algebra is continuous) in the affirmative for such Fréchet algebras.

In this paper we study the structure of topological algebras having an orthogonal Schauder basis. In $\S 1$ we discuss some properties of bases in topological algebras which we will use later. In $\S 2$ we describe the closed ideals and show that each closed ideal is the closure of the linear span of the basis elements it contains. In $\S 3$ we give a characterization of complete locally $m$-convex algebras with identity having an orthogonal basis. In another paper [4] we study topological algebras having unconditional orthogonal bases.

For definitions and results concerning bases in Banach spaces see [1], [7]. For general notions regarding topological algebras see Michael [5] and Zelazko [8]. A sequence $\left\{x_{n}\right\}$ in a topological vector space $E$ is a basis if for each $x \in E$ there is a unique sequence of scalars $\left\{\alpha_{n}\right\}$ such that $x=\sum_{n=1}^{\infty} \alpha_{n} x_{n}$. Each linear functional $x_{n}^{*}(x)=$ $\alpha_{n}$ is called a coefficient functional. If each $x_{n}^{*}$ is continuous then $\left\{x_{n}\right\}$ is called a Schauder basis. It is well known that each basis in a complete metrizable vector space is a Schauder basis. We show that each orthogonal basis in a locally $m$-convex algebra is a Schauder basis (Prop. 3.1) and each unital locally $m$-convex algebra $A$ with an orthogonal basis is metrizable (Theorem 3.3) and if, in addition 
$A$ is complete, then it is isomorphic and homeomorphic with the Fréchet algebra $s$ of all complex sequences (Theorem 3.4). These results generalize results in [3] proved for Fréchet algebras.

The authors are very grateful to the referee for pointing out an error and suggesting many improvements.

1. Orthogonal bases. In this section we consider the following conditions on a topological algebra $A$ with a Schauder basis $\left\{x_{n}\right\}$ :

(i) $x_{n} x_{m}=0$ for $n \neq m$;

(ii) $x_{n} x_{m}=0$ for $n \neq m$ and $x_{n}^{2} \neq 0$;

(iii) $x_{n} x_{m}=0$ for $n \neq m$ and $x_{n}^{2}=c_{n} x_{n}, c_{n} \neq 0$;

(iv) $x_{n} x_{m}=\delta_{n m} x_{n}$.

In the sequel a basis satisfying the condition (iv) will be called an orthogonal basis. We start with some elementary results.

It is obvious that (iv) $\Rightarrow$ (iii) $\Rightarrow$ (ii) $\Rightarrow$ (i). (ii) and (iii) are trivially equivalent. (iii) implies that one can replace $\left\{x_{n}\right\}$ by another base $\left\{y_{n}\right\}$ satisfying (iv). If $A$ has an identity, then (i) $\Rightarrow$ (ii). Thus for a topological algebra with an identity for a base $\left\{x_{n}\right\}$ to be orthogonal, it is enough to assume that $\left\{x_{n}\right\}$ satisfies (i) because we can always replace $\left\{x_{n}\right\}$ by another basis $\left\{y_{n}\right\}$ which satisfies (iv). The proofs of these statements as well as that of the following are easy and therefore omitted.

LEMMA 1.1. Let $A$ be a topological algebra satisfying (i).

(a) If $x=\sum \alpha_{i} x_{i}, y=\sum \beta_{i} x_{i}$, then $x y=\sum \alpha_{i} \beta_{i} x_{i}^{2}$.

Hence $A$ is commutative.

(b) If $x_{n}^{2}=x_{n}$ then the corresponding coefficient functional $x_{n}^{*}$ is multiplicative.

To describe the radical of a topological algebra with a basis satisfying (i), we first have the following:

LEMMA 1.2. Let $A$ be a topological algebra with a Schauder basis $\left\{x_{n}\right\}$ satisfying (i) and let $D$ be any subset of $\left\{x_{n}\right\}$. Then, $x=$ $\sum \alpha_{i} x_{i}$ belongs to $\overline{S p D}$ (closure of the linear span $S p D$ of $D$ ) iff $\alpha_{n}=0$ whenever $x_{n} \notin D$.

Proof. Suppose that for some $n \in N, \alpha_{n} \neq 0$ and $x_{n} \notin D$. Let $x \in \overline{S p D}$, then there is a net $\left\{x_{\lambda}\right\}$ in $S p D$ such that $x_{\lambda} \rightarrow x$. Since each coordinate functional $x_{n}^{*}$ is continuous, we have $0=\lim _{\lambda} x_{n}^{*}\left(x_{\lambda}\right)=$ $x_{n}^{*}(x)=\alpha_{n} \neq 0$, a contradiction. Conversely, if $x=\sum \alpha_{i} x_{i}$ and $\alpha_{n}=$ 0 whenever $x_{n} \notin D$, then clearly $S_{k}(x)=\sum_{i=1}^{k} \alpha_{i} x_{i} \in S p D$ for all $k \in N$. Whence we have $x=\lim _{k \rightarrow \infty} S_{k}(x) \in \overline{S p D}$. 
LEMMA 1.3. Let $A$ be a topological algebra with a Schauder basis $\left\{x_{n}\right\}$ satisfying (i). The following are equivalent:

(a) $x_{n} \in \operatorname{Rad} A$.

(b) $x_{n}^{2} \in \overline{S p}\left\{x_{k}: x_{k}^{2}=0\right\}$.

(c) $x_{n}^{*}\left(x_{n}^{2}\right)=0$.

(d) $x_{n}^{3}=0$.

Proof. Let $x_{n}^{2}=\sum_{k=1}^{\infty} \alpha_{k} x_{k}$, then $0=x_{k} x_{n}^{2}=\alpha_{k} x_{k}^{2}$ and so $\alpha_{k}=0$ whenever $x_{k}^{2} \neq 0$. Thus, by Lemma 1.2, $\alpha_{n}=x_{n}^{*}\left(x_{n}^{2}\right)=0$ iff $x_{n}^{2} \in$ $\overline{S p}\left\{x_{k}: x_{k}^{2}=0\right\}$. This proves $(\mathrm{b}) \Leftrightarrow(\mathrm{c})$. For $(\mathrm{c}) \Longrightarrow(\mathrm{d})$, note that

$$
x_{n}^{3}=x_{n} x_{n}^{2}=x_{n}\left(\sum_{k=1}^{\infty} x_{k}^{*}\left(x_{n}^{2}\right) x_{k}\right)=x_{n}^{*}\left(x_{n}^{2}\right) x_{n}^{2}=0 .
$$

Since $(d) \Rightarrow$ (a) is obvious, it remains to show that $(a) \Rightarrow(b)$. Sup-

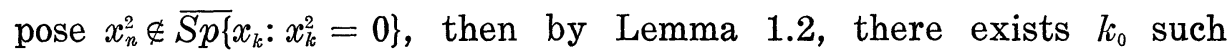
that $\alpha_{k_{0}} \neq 0$ and $x_{k_{0}}^{2} \neq 0$. By the first sentence in this proof $k_{0}=n$. Thus $\alpha_{n} \neq 0$, whence the sequence $\left\{y_{k}\right\}$ where $y_{k}=x_{k}, k \neq n$, and $y_{n}=x_{n} / \alpha_{n}$, is a basis for $A$, and $y_{n}^{2}=x_{n}^{2} / \alpha_{n}^{2}=\sum_{k=1}^{\infty}\left(\alpha_{k} / \alpha_{n}^{2}\right) x_{k}$, so $y_{n}^{*}\left(y_{n}^{2}\right)=$ 1 and $y_{n}^{*}\left(y_{k}^{2}\right)=0$ for $k \neq n$. Now, for

$$
\begin{aligned}
x, y \in A, y_{n}^{*}(x y) & \\
& =y_{n}^{*}\left(\sum_{k=1}^{\infty} y_{k}^{*}(x) y_{k}^{*}(y) y_{k}^{2}\right)=y_{n}^{*}(x) y_{n}^{*}(y) y_{n}^{*}\left(y_{n}^{2}\right)=y_{n}^{*}(x) y_{n}^{*}(y) .
\end{aligned}
$$

Thus $y_{n}^{*}$ is a continuous multiplicative linear functional and $y_{n}^{*}\left(y_{n}\right)=$ $1 \neq 0$. It follows that $y_{n} \notin \operatorname{Rad} A$, hence the same is true for $x_{n}$.

REMARK. From the above proof we note that in general, for a basis element $x_{n}$, either $x_{n}^{3}=0$ or $x_{n}^{3}=c_{n} x_{n}^{2}, \alpha \in C$. By a suitable transformation $\left\{x_{n}\right\}$ can be "normalized" to a basis $\left\{y_{n}\right\}$ so that $y_{n}^{3}=$ $y_{n}^{2}$ and by the above proof $y_{n}^{*}$ is then multiplicative.

THEOREM 1.4. $\operatorname{Rad} A=S p\left\{x_{n}: x_{n}\right.$ satisfies one of the equivalent conditions in Lemma 1.3\}. In particular, $\operatorname{Rad} A=\left\{x \in A: x^{3}=0\right\}$.

Proof. Let $D=\left\{x_{n}: x_{n}^{3}=0\right\}$. We show $\operatorname{Rad} A=\overline{S p D}$. To this end let $x \in \overline{S p D}, x=\sum \alpha_{k} x_{k}$. By Lemma 1.2, $\alpha_{k} \neq 0$ iff $x_{k}^{3}=0$, so $x^{3}=\sum_{k=1}^{\infty} \alpha_{k}^{3} x_{k}^{3}=0$, hence $x \in \operatorname{Rad} A$. Conversely, if $x \notin \overline{S p D}$, then there exists $k_{0}$ such that $\alpha_{k_{0}} \neq 0$ and $x_{k_{0}}^{3} \neq 0$. By choosing a basis as in $(a) \Rightarrow(b)$ of Lemma 1.3 , we show that $x \notin \operatorname{Rad} A$.

COROLlaRY 1.5. A topological algebra with an orthogonal Schauder basis is semisimple. 
Corollary 1.6. An F-algebra with an unconditional (see [1]) orthogonal basis has unique $F$-algebra topology.

Proof. By Theorem 4 of [3], $A$ is functionally continuous, commutative (Lemma 1.1) and semisimple (Corollary 1.5). By a Theorem of Michael [5, p. 62], $A$ has unique $F$-algebra topology.

Proposition 1.7. If $A$ is a topological algebra with a Schauder basis satisfying (i), then $A / \operatorname{Rad} A$ has an orthogonal basis.

Proof. If $D$ is any subset of $\left\{x_{i}\right\}$ and $\left\{x_{i_{k}}\right\}$ is the sequence of basis elements complementary to $D$, then the sequence $\left\{\eta\left(x_{i_{k}}\right)\right\}$, where $\eta: A \rightarrow A / \overline{S p D}$ is the canonical map, is a basis for $A / \overline{S p D}$. (This is proved in [7, Prop. 4.1] for Banach spaces but the theorem is true for Schauder bases in any TVS by a slight modification of the proof there). Now, if $D$ is as in the proof of Theorem 1.4, then $\operatorname{Rad} A=$ $\overline{S p D}$ and a simple verification shows that the basis $\left\{\eta\left(x_{i_{k}}\right)\right\}$ can be modified to yield an orthogonal basis for $A / \operatorname{Rad} A$.

We end this section by showing that an orthogonal basis in a topological algebra $A$ is "essentially unique". Precisely we have:

THEOREM 1.8. If $\left\{x_{i}\right\}$ and $\left\{y_{i}\right\}$ are orthogonal bases in a topological algebra $A$, then $\left\{x_{i}\right\}=\left\{y_{i}\right\}$.

Proof. Let $x_{n} \in\left\{x_{i}\right\}$. There exists $y_{m} \in\left\{y_{i}\right\}$ such that $x_{n} y_{m} \neq 0$. For, otherwise it follows that $x_{n}=0$, which is impossible. Now writing $x_{n}=\sum \alpha_{i} y_{i}$ and multiplying it by $y_{m}$, we obtain $x_{n} y_{m}=\alpha_{m} y_{m}$ which, if multiplied by $x_{n}$, yields $x_{n} y_{m}=\alpha_{m} x_{n} y_{m}$. This implies that $\alpha_{m}=1$ and so $x_{n} y_{m}=y_{m}$. Now writing $y_{m}=\sum \beta_{i} x_{i}$, by similar arguments we get $x_{n} y_{m}=x_{n}$, whence $x_{n}=y_{m}$. This proves that $\left\{x_{i}\right\} \subset\left\{y_{i}\right\}$ and the result follows by symmetry.

2. Closed ideals. Throughout this section $A$ will denote a topological algebra with an orthogonal Schauder basis $\left\{x_{n}\right\}$. Also for each $x_{n}^{*}$, let $M_{n}=\left\{x \in A: x_{n}^{*}(x)=0\right\}$ be its kernel.

THEOREM 2.1. If $I$ is a closed ideal in $A$, then there exists $n \in$ $N$ such that $I \subseteq M_{n}$. In particular, $\left\{M_{n}: n \in N\right\}$ is the set of all closed maximal ideals of $A$ and this set with the Gelfand Topology is homeomorphic to $N$.

Proof. If $I \subseteq M_{n}, n \geqq 1$, then for each $n \in N$, there exists $x \in I$ with $x_{n}^{*}(x) \neq 0$. Since $x_{n}^{*}(x)^{-1} x_{n} x=x_{n}$ we have $x_{n} \in I$ for all $n \geqq 1$. Thus $I$ is dense in $A$, contradicting the assumption that $I$ is closed. 
To see that this set is discrete, consider the subbasic neighborhood $V$ of $x_{n}^{*}$,

$$
V=V\left(\frac{1}{2}, x_{n}, x_{n}^{*}\right)=\left\{x_{k}^{*}:\left|x_{k}^{*}\left(x_{n}\right)-x_{n}^{*}\left(x_{n}\right)\right| \leqq \frac{1}{2}\right\}=\left\{x_{n}^{*}\right\},
$$

the last equality being true because $x_{k}^{*}\left(x_{n}\right)=\delta_{k n}$.

For $I \subset A$, set $Z(I)=\left\{n \in N: x_{n}^{*}(x)=0\right.$ for all $\left.x \in I\right\}$ and write $Z(x)$ for $Z(\{x\})$. Also let $K=\left\{n: x_{n} \in I\right\}$. With this notation Lemma 1.2 says that $x \in \overline{S p}\left\{x_{n}: n \in K\right\}$ iff $N \backslash K \subseteq Z(x)$.

THEOREM 2.2. Let $I$ be a closed ideal in A. Then

(a) $Z(I)=N \backslash K$.

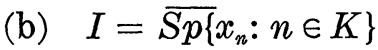

$$
\begin{aligned}
& =\cap\left\{M_{n}: n \in Z(I)\right\} \\
& =\{x \in A: Z(I) \leqq Z(x)\} .
\end{aligned}
$$

Proof. (a) If $n \in Z(I)$, then $x_{n}^{*}(x)=0$ for all $x \in I$. Thus $x_{n} \notin I$ and so $n \notin K$. Conversely, if $n \notin Z(I)$, then $x_{n}^{*}(x) \neq 0$ for some $x \in I$. Now $x_{n} x=x_{n}^{*}(x) x_{n}$, so $x_{n} \in I$, whence $n \in K$.

(b) Since $I$ is closed, $\overline{S p}\left\{x_{n}: n \in K\right\} \subset I$. If $x \in I$, then by (a), $N \backslash K \subseteq Z(x)$ and so by Lemma 1.2, $x \in \overline{S p}\left\{x_{n}: n \in K\right\}$. The other two equalities follow from this and Lemma 1.2.

REMARK. In view of the proof of Proposition 1.7, the first equality of part (b) of the above proposition shows that for any closed ideal $I$ of $A, A / I$ has an orthogonal basis.

CoRollary 2.3. Let $x \in A$ and let $I=\overline{\langle x\rangle}$, the closure of the principle ideal generated by $x$. Then

(a) $Z(I)=\left\{n: x_{n}^{*}(x)=0\right\}$.

(b) $I=\overline{S p}\left\{x_{n}: n \in N \backslash Z(I)\right\}$

$$
\begin{aligned}
& =\cap\left\{M_{n}: n \in Z(I)\right\} \\
& =\{y \in A: Z(x) \subset Z(y)\} .
\end{aligned}
$$

Proof. The ideal $\langle x\rangle$ contains exactly those basis elements $x_{n}$ for which $x_{n}^{*}(x) \neq 0$. Whence $\overline{\langle x\rangle}$ contains exactly those same basis elements also. Now (a) follows from part (a) of Theorem 2.2. Part (b) follows directly from Theorem 2.2(b).

3. Locally $M$-convex algebras. In this section we generalize some results in [3]. In particular we give a characterization of complete locally $m$-convex algebras with an orthogonal basis. 
Proposition 3.1. Each orthogonal basis in a locally m-convex algebra (cf:[5]) $A$ is a Schauder basis.

Proof. Let $\left\{x_{n}\right\}$ be an orthogonal basis in $A$ and let $\left\{p_{\alpha}\right\}$ be a family of submultiplicative seminorms generating the topology of $A$. For $x \in A, x=\sum x_{n}^{*}(x) x_{n}$ we have $x x_{n}=x_{n}^{*}(x) x_{n}^{2}=x_{n}^{*}(x) x_{n}(n \geqq 1)$ and so for each $p_{\alpha}$ and $n,\left|x_{n}^{*}(x)\right| p_{\alpha}\left(x_{n}\right) \leqq p_{\alpha}(x) p_{\alpha}\left(x_{n}\right)$. Since $A$ is Hausdorff, there exists $p_{\beta}$ such that $p_{\beta}\left(x_{n}\right) \neq 0$, with this $p_{\beta}$ from the above inequality we get $\left|x_{n}^{*}(x)\right| \leqq p_{\beta}(x), x \in A$, which proves the continuity of $x_{n}^{*}$ for each $n \geqq 1$.

REMARK. Note that if $f$ is any multiplicative linear functional on $A$ with $f\left(x_{n}\right) \neq 0$ for some $n \geqq 1$, then by the arguments used in the proof of Theorem 2.1 we get that $f=x_{n}^{*}$. Hence $f$ is continuous by the above proposition. This is known for Fréchet algebras [3].

Let $E$ be a topological vector space with a basis $\left\{x_{n}\right\}$.' We define a map $\sigma$ from $E$ into the space $s$ of all complex sequences by $\sigma(x)=\left\{x_{n}^{*}(x)\right\}_{n=1}^{\infty}, x \in A$.

LEMMA 3.2. Let $A$ be a locally m-convex algebra with an orthogonal basis $\left\{x_{n}\right\}$ and let $P$ be a family of submultiplicative seminorms generating the topology of $A$. Consider the following statements:

(a) $\sigma: A \rightarrow s$ is surjective.

(b) A has an identity.

(c) For each $p \in P$ there exists $N \in N$ such that $p\left(x_{n}\right)=0$ whenever $n>N$.

Then (1): (a) implies each of (b) and (c), and (b) implies (c); (2): if $A$ is complete, these statements are equivalent.

Proof. The proof of this lemma follows from the proofs of Propositions 1 and 3 of [3] if one replaces the sequence of seminorms by a family of seminorms.

THEOREM 3.3. Let $A$ be a locally m-convex algebra with an orthogonal basis $\left\{x_{n}\right\}$. If $A$ has an identity, then $A$ is metrizable.

Proof. For $p \in P$, let $K_{p}=\left\{n: p\left(x_{n}\right)=0\right\}$ and for $p, q \in P$ define $p R q$ iff $K_{p}=K_{q}$. Note that $R$ is an equivalence relation and since each set $K_{p}$ is cofinite in $N$ (Lemma 3.2) there can be at most a countable number of $R$-classes. Let $p R q$. Clearly $\operatorname{ker} p=\{x \in A$ : $p(x)=0\}$ is a closed ideal in $A$ and so by Theorem 2.2(b), $\operatorname{ker} p=$ $\overline{S p}\left\{x_{n}: n \in K_{p}\right\}$ from which it follows that $\operatorname{ker} p=\operatorname{ker} q$ and is of 
finite codimension in $A$. Thus $A / \operatorname{ker} p=A / \operatorname{ker} q$ is finite dimensional so $p$ and $q$ induce equivalent norms on it. Hence $p$ and $q$ are equivalent seminorms on $A$.

We have the following theorem which generalizes Theorem 1 of [3].

THEOREM 3.4. Let $A$ be a complete locally m-convex algebra with an orthogonal basis $\left\{x_{n}\right\}$ and let $P$ be a family of seminorms generating the topology of $A$. The following are equivalent:

(a) A has an identity.

(b) $\sigma$ is onto $s$.

(c) for every $p \in P, p\left(x_{n}\right)=0$ for all sufficiently large $n$.

(d) $A$ is algebraically and topologically isomorphic to $s$.

4. Examples. We conclude this paper by giving several examples.

EXAMPLE 1. The Banach algebras $l^{p}(\boldsymbol{N})=\left\{\left\{\alpha_{i}\right\} \in s: \sum\left|\alpha_{i}\right|^{p}<\infty\right\}$ $1 \leqq p<\infty ; c_{0}$, the algebra of complex sequences converging to 0 , and the Fréchet algebra $s$ of all complex sequences (all with pointwise operations) have the sequence $e_{n}=\left(\delta_{n m}\right)_{m=0}^{\infty}, n \geqq 1$ as a basis. Clearly this basis is orthogonal in our sense.

ExAmple 2. The space $L^{p}(T), 1<p<\infty$ is a Banach algebra with convolution multiplication (see [8]). The sequence of trigonometric polynomials $e_{n}(t)=t^{n}, t \in T, n \geqq 1$, is an orthogonal basis for $L^{p}(T)$, where $T$ is the circle group.

ExAmple 3. The Hardy spaces $H^{p}(D), 1<p<\infty$, where $D$ is the open unit dise in $C$ are Banach algebras with the product

$$
(f * g)(x)=\frac{1}{2 \pi i} \int_{|z|=r} f(z) g\left(x z^{-1}\right) z^{-1} d z,
$$

where $f, g \in H^{p}$ and $|x|<r<1$ [6]. The sequence $e_{n}(x)=x^{n}, x \in D$, is a basis for $H^{p}$ and a simple computation shows that it is an orthogonal basis with respect to the above product.

Let $E$ be a Banach space with an unconditional basis $\left\{x_{i}\right\}$. For $x, y \in E, x=\sum \alpha_{i} x_{i}, y=\sum \beta_{i} x_{i}$, define $x * y=\sum \alpha_{i} \beta_{i} x_{i}$. This definition makes sense because, without loss of generality, assume that the basis $\left\{x_{i}\right\}$ is normalized (i.e., $\left\|x_{i}\right\|=1, i \geqq 1$ ). Then $\lim _{i \rightarrow \infty} \alpha_{i}=$ 0 [6]. Thus the sequence $\left\{\alpha_{i}\right\}$ is bounded and therefore, since $\sum \beta_{i} y_{i}$ converges unconditionally (hence is bounded multiplier convergent [1]), it follows that $\sum \alpha_{i} \beta_{i} x_{i}$ converges in $E$. Thus $x^{*} y$ is a well defined element of $E$ for $x, y \in E$. Moreover, it is clear that $A$ is 
an algebra with this product. More is true:

Proposition 4.1. If $E$ is a Banach space with an unconditional basis, then $E$ is a Banach algebra (in an equivalent norm) with *product and the basis is orthogonal.

Proof. Without loss of generality assume that the basis is normalized and let $\|\cdot\|_{0}$ be the norm on $E$ given by $\|x\|_{0}=$ $\sup _{n \in N}\left|x_{n}^{*}(x)\right|$. Then with the map $\sigma: E \rightarrow m$ (the Banach space of bounded sequence with the sup norm $\|\cdots\|_{\infty}$ ) defined by $\sigma(x)=$ $\left\{x_{n}^{*}(x)\right\}_{n=1}^{\infty}$, we have for each $f_{k}$, the $k$ th coefficient functional on $m$,

$$
\left(f_{k}^{\circ} \circ \sigma\right)(x)=f_{k}(\sigma(x))=f_{k}\left(\left\{x_{n}^{*}(x)\right\}_{n=1}^{\infty}\right)=x_{k}^{*}(x) .
$$

Since $E$ is a Banach space, the functionals $x_{n}^{*}$ are continuous [7]. Hence each $f_{k} \circ \sigma$ is continuous on $E$ for each $k \geqq 1$. Since the family $\left\{f_{k}\right\}$ is a separating family of continuous linear functionals on $m$, it follows by the closed graph theorem [2] that $\sigma$ is continuous. Therefore, there exists $c_{1}>0$ such that $\|\sigma(x)\|_{\infty} \leqq c_{1}\|x\|, x \in E$. Since $\|x\|_{0}=\|\sigma(x)\|_{\infty}$, we have $\|x\|_{0} \leqq c_{1}\|x\|$. Now define

$$
\|x\| \mid=\sup _{f \in E^{\prime},\|f\| \leq 1}\left[\sum_{n=1}^{\infty}\left|x_{n}^{*}(x)\right|\left|f\left(x_{n}\right)\right|\right],
$$

where $E^{\prime}$ is the topological dual of $E$. It is easy to show [7, p. $463]$ that ||$\cdots \mid \|$ is a norm on $E$ equivalent to the original norm of $E$. Hence, there is a constant $c_{2}>0$ such that $\|x\| \leqq c_{2}\|x\|, x \in$ E. Now

$$
\begin{aligned}
\|x * y\| & =\sup _{f \in E^{\prime},\|f\| \leqq 1}\left[\sum_{n=1}^{\infty}\left|x_{n}^{*}(x) x_{n}^{*}(y) \| f\left(x_{n}\right)\right|\right] \\
& \leqq\|x\|_{0} \sup _{f \in E^{\prime},\|f\| \leqq 1}\left[\sum_{n=1}^{\infty}\left|x_{n}^{*}(y) \| f\left(x_{n}\right)\right|\right] \\
& \leqq c_{1}\|x\|\|y\| \mid \\
& \leqq c_{1} c_{2}\|x\|\|\| y \| .
\end{aligned}
$$

This shows that $E$ is a Banach algebra in a norm equivalent to

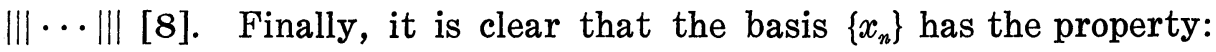
$x_{n} * x_{m}=\delta_{n m} x_{n}, n, m \geqq 1$.

REMARK. We note here that an infinite dimensional normed algebra $A$ with an orthogonal basis $\left\{x_{n}\right\}$ cannot have an identity $e$. For, if $e \in A, e=\sum_{k=1}^{\infty} x_{k}$ converges, hence $\left\|x_{n}\right\|<1$ for sufficiently large $n$. Thus, $\left\|x_{n}\right\|=\left\|x_{n}^{k}\right\| \leqq\left\|x_{n}\right\|^{k}$ for all $k \geqq 1$. So, $\left\|x_{n}\right\|=0$ which is impossible since $x_{n} \neq 0$.

We now give an example of a topological algebra with an 
orthogonal basis which is not a Banach algebra.

EXAMPLE 4. Let $H(D)$ be the $F$-space (with the compact-open topology) of all functions holomorphic in the open unit disc $D . H(D)$ is a topological algebra with identity 1 with the product

$$
(f * g)(x)=\frac{1}{2 \pi i} \int_{|z|=r} f(z) g\left(x z^{-1}\right) z^{-1} d z,
$$

where $x \in D$, and $|x|<r<1[6]$. The sequence of functions $\xi_{n}(z)=$ $z^{n}, z \in D, n \geqq 0$ is a basis for $H(D)$. A simple computation shows that this basis is an orthogonal basis. Note that $H(D)$ cannot be locally $m$-convex in view of Theorem 3.4, since it is not $s$.

Finally, we note that if $A$ and $B$ are topological algebras with orthogonal bases then the product basis [7, p. 28] in $A \times B$ is an orthogonal basis as can easily be checked (note that the construction of product bases for Banach spaces given in [7] has a natural extension to topological vector spaces and the proofs are similar to the Banach space case). Also, if $A$ and $B$ are Banach algebras with orthogonal bases then it is easy to check that the tensor product of these bases [7, p. 173] in $A \otimes_{p} B$, the projective tensor product of $A$ and $B$, is an orthogonal basis for the Banach algebra $A \otimes_{p} B$.

\section{REFERENCES}

1. M. M. Day, Normed Linear Spaces, Academic Press Inc., N. Y., 1962.

2. T. Husain, The open mapping and closed graph theorems in topological vector spaces, Oxford Math. Monographs, 1965.

3. T. Husain and J. Liang, Multiplicative functionals on Fréchet algebras with bases, Canad. J. Math., 29 (1977), 270-276.

4. T. Husain and S. Watson, Unconditional orthogonal bases, Proc. Amer. Math. Soc. 79 (1980), 539-545.

5. A. E. Michael, Locally multiplicatively convex topological algebras, Amer. Math. Soc., Memoirs, 11 (1952).

6. P. Procelli, Linear Space of Analytic Functions, Rand McNally, Chicago, 1966.

7. I. Singer, Bases in Banach Spaces I, Springer-Verlag, N. Y. Heidelberg-Berlin, 1970.

8. W. Zelazko, Banach Algebras, Elsevier, Amsterdam, 1973.

Received May 17, 1979 and in revised form April 18, 1980. The work by the first author was supported by an NSERC Grant.

McMaster University

Hamilton, Ontario

CanAda L8S $4 \mathrm{~K} 1$ 



\section{PACIFIC JOURNAL OF MATHEMATICS}

\section{EDITORS}

DONALD BABBITT (Managing Editor)

University of California

Los Angeles, CA 90024

Hugo RossI

University of Utah

Salt Lake City, UT 84112

C. C. MOORE and ANDREW OGG

University of California

Berkeley, CA 94720

\section{J. DugundjI}

Department of Mathematics

University of Southern California

Los Angeles, CA 90007

R. FinN and J. Milgram

Stanford University

Stanford, CA 94305

\section{ASSOCIATE EDITORS}
E. F. BeCKENBACH
B. H. Neumann
F. WoLF
K. YoSHIDA

\section{SUPPORTING INSTITUTIONS}

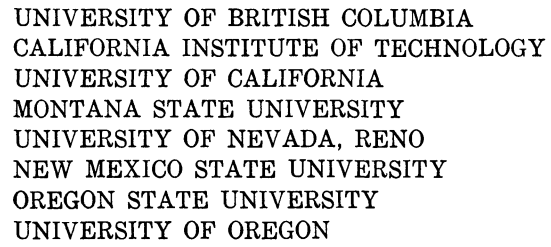

UNIVERSITY OF BRITISH COLUMBIA CALIFORNIA INSTITUTE OF TECHNOLOGY UNIVERSITY OF CALIFORNIA MONTANA STATE UNIVERSITY UNIVERSITY OF NEVADA, RENO NEW MEXICO STATE UNIVERSITY OREGON STATE UNIVERSITY UNIVERSITY OF OREGON

\author{
UNIVERSITY OF SOUTHERN CALIFORNIA \\ STANFORD UNIVERSITY \\ UNIVERSITY OF HAWAII \\ UNIVERSITY OF TOKYO \\ UNIVERSITY OF UTAH \\ WASHINGTON STATE UNIVERSITY \\ UNIVERSITY OF WASHINGTON
}

The Supporting Institutions listed above contribute to the cost of publication of this Journal, but they are not owners or publishers and have no responsibility for its content or policies.

Mathematical papers intended for publication in the Pacific Journal of Mathematics should be in typed form or offset-reproduced, (not dittoed), double spaced with large margins. Please do not use built up fractions in the text of the manuscript. However, you may use them in the displayed equations. Underline Greek letters in red, German in green, and script in blue. The first paragraph or two must be capable of being used separately as a synopsis of the entire paper. Please propose a heading for the odd numbered pages of less than 35 characters. Manuscripts, in triplicate, may be sent to any one of the editors. Please classify according to the scheme of Math. Reviews, Index to Vol. 39. Supply name and address of author to whom proofs should be sent. All other communications should be addressed to the managing editor, or Elaine Barth, University of California, Los Angeles, California, 90024.

50 reprints to each author are provided free for each article, only if page charges have been substantially paid. Additional copies may be obtained at cost in multiples of 50 .

The Pacific Journal of Mathematics is issued monthly as of January 1966. Regular subscription rate: $\$ 84.00$ a year (6 Vols., 12 issues). Special rate: $\$ 42.00$ a year to individual members of supporting institutions.

Subscriptions, orders for numbers issued in the last three calendar years, and changes of address shoud be sent to Pacific Journal of Mathematics, P.O. Box 969, Carmel Valley, CA 93924, U.S.A Old back numbers obtainable from Kraus Periodicals Co., Route 100, Millwood, NY 10546.

PUBLISHED BY PACIFIC JOURNAL OF MATHEMATICS, A NON-PROFIT CORPORATION

Printed at Kokusai Bunken Insatsusha (International Academic Printing Co., Ltd.). 8-8, 3-chome, Takadanobaba, Shinjuku-ku, Tokyo 160, Japan. 


\section{Pacific Journal of Mathematics}

\section{Vol. 91, No. 2 December, 1980}

Victor P. Camillo and Julius Martin Zelmanowitz, Dimension modules ... . . 249

Yonina S. Cooper, Stable sequences in pre-abelian categories ........... 263

Chandrakant Mahadeorao Deo and H. Ship-Fah Wong, On Berry-Esseen approximation and a functional LIL for a class of dependent random fields.........................................

H. P. Dikshit and S. N. Dubey, $|C, 1|$ summability of series associated with

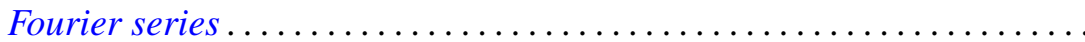

M. Edelstein, On the homomorphic and isomorphic embeddings of a semiflow into a radial flow.

Gilles Godefroy, Compacts de Rosenthal ..................... 293

James Guyker, Commuting hyponormal operators ................ 307

Thomas Eric Hall and Peter R. Jones, On the lattice of varieties of bands of

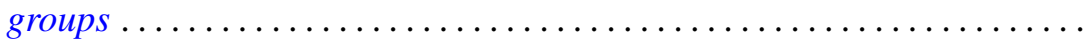

Taqdir Husain and Saleem H. Watson, Topological algebras with orthogonal Schauder bases ....................................

V. K. Jain, Some expansions involving basic hypergeometric functions of two variables. . .

Joe W. Jenkins, On group actions with nonzero fixed points ........... 363

Michael Ellsworth Mays, Groups of square-free order are scarce ........ 373

Michael John McAsey, Canonical models for invariant subspaces... 377

Peter A. McCoy, Singularities of solutions to linear second order elliptic partial differential equations with analytic coefficients by approximation methods...

Terrence Millar, Homogeneous models and decidability.

Stephen Carl Milne, A multiple series transformation of the very well poised

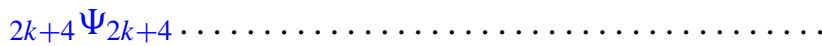

Robert Olin and James E. Thomson, Irreducible operators whose spectra are spectral sets...

Robert John Piacenza, Cohomology of diagrams and equivariant singular

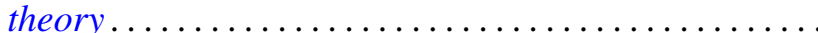

Louis Jackson Ratliff, Jr., Integrally closed ideals and asymptotic prime divisors

Robert Breckenridge Warfield, Jr., Cancellation of modules and groups and stable range of endomorphism rings.................

B. J. Day, Correction to: "Locale geometry" ...............

Stanley Stephen Page, Correction to: "Regular FPF rings" ... 